\title{
Correlação entre perda auditiva e resultados dos questionários Hearing Handicap Inventory for the Adults - Screening Version HHIA-S e Hearing Handicap Inventory for the Elderly - Screening Version - HHIE-S
}

\author{
Correlation between hearing loss and the results of the following questionnaires: Hearing \\ Handicap Inventory for the Adults - Screening Version HHIA-S and Hearing Handicap \\ Inventory for the Elderly - Screening Version - HHIE-S
}

\author{
Isabela Hoffmeister Menegotto', Cristina Loureiro Chaves Soldera², Paula Anderle3 ${ }^{3}$ Tanise Cristaldo Anhaia4. \\ 1) Doutora em Distúrbios da Comunicação Humana pela Universidade Federal de São Paulo. Professora do Departamento de Fonoaudiologia da UFCSPA. \\ 2) Doutoranda em Gerontologia Biomédica (PUCRS). Professora do Departamento de Fonoaudiologia da UFCSPA. \\ 3) Especializanda em Motricidade orofacial pelo CEFAC-RS. Fonoaudióloga Clínica. \\ 4) Mestranda do PPG em Ciências da Reabilitação da UFCSPA. Mestranda do PPG em Ciências da Reabilitação da UFCSPA. \\ Instituição: Universidade Federal de Ciências da Saúde de Porto Alegre. \\ Porto Alegre / RS - Brasil. \\ Endereço para correspondência: Tanise Cristaldo Anhaia - Rua Duque de Caxias, 203/22 - Porto Alegre / RS - Brasil - CEP: $90010-282$ - Telefone: (+55 51) 9287-4589 \\ -E-mail: tanise.anhaia@bol.com.br \\ Artigo recebido em 25 de Março de 2011. Artigo aprovado em 19 de Abril de 2011
}

\section{RESUMO}

Introdução: Os questionários de autoavaliação são úteis para quantificar as consequências emocionais e sociais/situacionais percebidas em função da perda de audição, podendo ser utilizados em diversas situações na rotina clínica, como a triagem auditiva.

Objetivo: Verificar a sensibilidade e a especificidade dos questionários HHIA-S e HHIE-S na detecção de perda auditiva e suas aplicabilidades em triagens auditivas e analisar a capacidade desses questionários em detectar diferentes graus de comprometimento auditivo na população estudada.

Método: Estudo retrospectivo, 51 indivíduos, entre 18 e 88 anos, responderam aos questionários Hearing Handicap Inventory for Adults Screening Version - HHIA-S e Hearing Handicap Inventory for the Elderly Screening Version - HHIES em sala de espera de um ambulatório de otorrinolaringologia do SUS

Resultados: Os instrumentos revelaram baixa sensibilidade (47\%), não identificando indivíduos com perda auditiva; porém, apresentaram alta especificidade (75\%), identificando, corretamente, indivíduos que não apresentavam problemas de audição. Ainda, não existiu associação significativa entre o grau da perda auditiva e o grau de restrição de participação. Conclusão: Os referidos questionários apresentaram baixa sensibilidade e alta especificidade, não sendo eficazes para triagens auditivas em um grupo com queixas auditivas prévias, e também não foram capazes de detectar diferentes tipos e graus de comprometimento auditivo.

Palavras-chave: questionários, sensibilidade e especificidade, perda auditiva, envelhecimento.

\section{SUMMARY}

Introduction: The self-assessment questionnaires are useful to measure the emotional and social/transient consequences resulting from hearing loss, and they can be used in a wide range of situations in the clinical routine, such as auditory screening.

Objective: Check the sensitivity and specificities of HHIA-S and HHIE-S questionnaires to identify a hearing loss and their usages in auditory screenings, as well as analyze the ability of these questionnaires to detect different degrees of hearing impairments in the studied people.

Method: Retrospective study with 51 individuals aged between 18 and 88, who filled out the Hearing Handicap Inventory for Adults Screening Version - HHIA-S and Hearing Handicap Inventory for the Elderly Screening Version - HHIE-S questionnaires at the waiting room of the Brazilian Unified Health System (SUS)'s otorhinolaryngology infirmary.

Results: The instruments showed a low sensitivity (47\%), not identifying individuals with a hearing loss; however, they showed a high specificity (75\%), accurately identifying individuals with no hearing disorder. Moreover, no significant association between the degree of hearing loss and the constraint degree for participation was found.

Conclusion: the aforementioned questionnaires revealed a low sensitivity and a high specificity, proving to be ineffective for auditory screenings in a group with previous hearing complaints in addition to being unable to detect different types and degrees of hearing impairment.

Keywords: questionnaires, sensitivity and specificity, hearing loss, aging. 


\section{INTRODUÇÃO}

Segundo dados da OMS, no Brasil cerca de 2.250.000 habitantes são portadores de deficiência auditiva, o que corresponderia a 1,5\% da população (1). Para atender esta população, o Sistema Único de Saúde (SUS) criou o Serviço de Atenção à Saúde Auditiva, que compreende triagem e monitoramento da audição de neonatos, pré-escolares e escolares; diagnóstico de perda auditiva de crianças a partir de três anos de idade, de jovens e de adultos (trabalhadores e idosos), respeitando as especificidades nas avaliações exigidas para cada um desses segmentos (2).

A perda de audição pode ser considerada uma das mais devastadoras em relação ao convívio social do indivíduo. Em adultos, o impacto deste tipo de alteração auditiva pode associar-se ao declínio cognitivo, à depressão e à redução do estado funcional, principalmente para aqueles que apresentam a perda, mas não foram avaliados ou tratados (3).

Sendo assim, o levantamento dos indivíduos com dificuldade de audição de uma comunidade, sua localização e o estudo de suas condições sociais são de extrema importância para a adequação das medidas de saúde pública, nos vários níveis de prevenção (4). Para isso, tornam-se imprescindíveis métodos de triagem auditiva que tenham alta sensibilidade na detecção da perda auditiva em indivíduos que nem suspeitam ter alguma dificuldade de audição, e que, por isso, não procuram atendimento.

A triagem tem por definição a aplicabilidade em uma população numerosa, a rapidez e simplicidade na sua aplicação, e deve identificar os indivíduos que têm alta probabilidade de portar um distúrbio que está sendo testado (5). A triagem auditiva, por sua vez, deve ter alta sensibilidade e especificidade para identificar a presença de uma alteração auditiva quando esta alteração realmente existe, além de ter baixo custo (6).

O teste ouro para a perda auditiva, a audiometria tonal liminar, requer pessoal treinado, cabine acústica e equipamento específico, dificultando assim a sua execução em larga escala. Por outro lado, o uso de questionários, que tem alta sensibilidade na indicação da perda auditiva e são de administração rápida e barata, pode ser uma opção viável para triagem auditiva em grandes populações (7).

Os questionários de autoavaliação são úteis para quantificar as consequências emocionais e sociais/ situacionais percebidas em função da perda de audição, podendo ser utilizados em diversas situações na rotina clínica, como triagem auditiva, entrevista inicial, aconselhamento, candidatura, avaliação do benefício, uso e satisfação do indivíduo com o aparelho de amplificação sonora e avaliação da efetividade dos programas de reabilitação audiológica (8). São questionários utilizados para este fim os The Abbreviated Profile of Hearin Aid Benefit - APHAB, The Nursing Home Hearing Handicap- NHHI, The Hearing HandicapInventory for the Elderly: Screening Version - HHIE-S, The Hearing Handicap Inventory for Adults-HHIA, entre outros (9).

Especificamenteentre esses questionários, o Hearing Handicap Inventory for the Adults Screening Version HHIA-S e o Hearing Handicap Inventory for the Elderly Screening Version - HHIE-S, que são versões reduzidas, respectivamente, do Hearing HandicapInventory for the Adult-HHIA e do Screening Hearing HandicapInventory for the Elderly - HHIE, tem aplicação rápida e fácil compreensão, o que favorece seu uso em indivíduos idosos. Exatamente por isso, o questionário HHIE-S é recomendado pela American Speech-Language-Hearing Association (ASHA) como um instrumento para triagem auditiva $(8,10)$.

Estudos realizados no exterior por STEWART e colaboradores, em 2002 (10), e por Chang, Ho e CHOU, em 2009 (11), pesquisaram a validade dos questionários HHIE-S e HHIA-S em relação à percepção da perda auditiva em idosos e adultos. Esses estudos demonstraram que os questionários têm alta sensibilidade e especificidade na detecção da perda auditiva nessa população.

No Brasil, já existem estudos (8, 12, 13, 14, 15, 16, $17,18)$ mostrando a utilidade de questionários de restrição de participação na identificação de indivíduos com perda auditiva, e no Rio Grande do Sul, o questionário HHIE-S foi utilizado para avaliar o impacto subjetivo de um programa de reabilitação auditiva em idosos (19). Por outro lado, existem estudos verificando a relação entre a queixa e a presença de perda auditiva em idosos (20) e mostrando o valor preditivo, sensibilidade e especificidade da indagação simples a respeito da presença de perda auditiva (21) no Estado do Rio Grande do Sul.

No primeiro estudo, os indivíduos responderam a um questionário demográfico, no qual havia uma lista de problemas de saúde que incluía a perda auditiva. Dos 50 participantes, de ambos os sexos, constatou-se que somente 12 (24\%) tinham queixa específica de perda auditiva, apesar de 33 (66\%) apresentarem perdas auditivas de grau leve, moderado, severo e profundo, não evidenciando relação entre a queixa e a perda auditiva. No segundo, com amostra de 795 indivíduos de ambos os sexos e todas as faixas-etárias, 525 (66\%) pacientes apresentaram queixas de perda auditiva, $68(8,6 \%)$ tiveram outras queixas auditivas e $202(25,4 \%)$ demonstraram queixas não auditivas. Os resultados evidenciaram que a queixa de perda auditiva 
apresentou sensibilidade de $80,9 \%$, especificidade de $69,6 \%$, valor preditivo positivo de $86,5 \%$ e negativo de $60,4 \%$.

Apesar disso, o uso de questionários de restrição de participação mais abrangentes permite uma melhor compreensão das condições auditivas dos indivíduos do que a mera indagação a respeito de sua condição auditiva, tanto em adultos como em idosos.

O presente trabalho teve, assim, o objetivo deverificar a sensibilidade e a especificidade dos questionários HHIA-S e HHIE-S na detecção de perda auditiva e sua aplicabilidade em triagens auditivas em pacientes adultos e idosos, com base em uma amostra de indivíduos atendidos pelo SUS no Serviço de Audiologia do Ambulatório de Otorrinolaringologia doHospital Santa Clara-Complexo Hospitalar Santa Casa de Porto Alegre. Além disso, como objetivo secundário, o presente trabalho buscou analisar a capacidade dos referidos questionários em detectar diferentes graus de comprometimento auditivo na população estudada.

\section{MÉTODO}

A execução do trabalho foi aprovada pelo Comitê de Ética em Pesquisa da Irmandade da Santa Casa de Misericórdia de Porto Alegre (ISCMPA), por meio do protocolo de número 3292/10, de 10 de maio de 2010.

A coleta de dados foi realizada no período compreendido entre maio a setembro de 2010, três vezes por semana, no turno da tarde, tratando-se de um estudo de corte transversal. A amostra de conveniência foi formada por indivíduos que se encontravam aguardando para a realização de exames audiológicos no Ambulatório de Otorrinolaringologia do SUS do Hospital Santa Clara Complexo Hospitalar Santa Casa de Porto Alegre.

Para compor a amostra, os participantes obedeceram aos seguintes critérios de inclusão: saber ler e escrever, declarar estar apto e interessado em participar da pesquisa e ter idade superior a 18 anos. Inicialmente, os participantes assinaram o Termo de Consentimento Livre e Esclarecido, autorizando o uso dos dados coletados no estudo, de acordo com as normas estabelecidas pelo Comitê de Ética da ISCMPA. Após a assinatura do mesmo, os indivíduos com mais de 60 anos, ou seja, considerados idosos segundo o Ministério da Saúde (20), receberam o questionário HHIE$\mathrm{S}$, e os sujeitos com menos de 60 anos, responderam ao instrumento HHIA-S.

O questionário Hearing HandicapInventory forthe Elderly Screening Version - HHIE-S foi desenvolvido por VENTRY e WEINSTEIN (1982)e adaptado para o português por Wieselberg (1997), e o Hearing Handicap Inventory for
Adults Screening Version - HHIA-S foi traduzido e adaptado para o português por Almeida (1998). Estes instrumentos são compostos por dez perguntas divididas em cinco itens relacionados à escala social/situacional e outros cinco correspondentes à escala emocional. Estes instrumentos são adaptações reduzidas do Hearing HandicapInventory for the Elderly - HHIE e do Hearing Handicap Inventory forthe Adult-HHIA, sendo assim, são os únicos protocolos equivalentes para serem aplicados em diferentes populações de acordo com a faixa etária (16). Por esse motivo, os questionários foram agrupados para a análise dos dados.

A técnica selecionada para aplicação do questionário foi a "papel-lápis", ou seja, o indivíduo foi orientado a ler e responder sozinho ao questionário. No entanto, não foi possível utilizar este tipo de técnica com alguns participantes da amostra, sendo então aplicada à técnica "frente a frente", que é a aplicação oral do questionário pela entrevistadora apenas com a leitura dos itens, sem maiores explicações ou elaborações sobre os mesmos. Isso ocorreu por solicitação ou opção do próprio indivíduo, de acordo com o que lhe era mais conveniente, no momento da aplicação, normalmente devido a dificuldades visuais ou de leitura. Os participantes que responderam aos questionários na modalidade "papel-lápis" e na modalidade "frente a frente" foram, de início, analisados separadamente.

A possibilidade de respostas e a pontuação das mesmas no HHIA-S são idênticas às do HHIE-S. Foi solicitado aos usuários responder "sim" (4 pontos), "às vezes" ( 2 pontos) ou "não" (nenhum ponto) para cada questão, de acordo com o que julgaram ser mais adequado ao seu caso ou situação. A possibilidade de escore de ambos os questionários varia de 0 (nenhuma percepção de restrição de participação) a 40 (restrição máxima de participação). Tal como o proposto por Rosis, Souza e IóRIO (12), os indivíduos foram agrupados em três categorias: 08 pontos (sem percepção da restrição de participação); 1023 pontos (percepção leve a moderada) e 24-40 pontos (percepção significativa da restrição de participação).

A coleta das audiometrias foi realizada por meio do preenchimento de um formulário com os limiares das frequências de 250, 500, 1000, 2000, 3000, 4000, 6000 e 8000 Hz para via aérea, e 500, 1000, 2000 e 4000 Hz para via óssea, de ambas as orelhas. O exame audiométrico foi coletado no prontuário de cada paciente, após ser realizado de acordo com o procedimento habitual do estágio curricular do curso de Fonoaudiologia da UFCSPA, realizado no referido Ambulatório. Os equipamentos utilizados para realizar as audiometrias foram os audiômetros Interacoustics AD 227 ou Sibelmed AC 50-D.

Quanto ao tipo de perda auditiva, foi utilizada a classificação de Silman e Silverman (22), a qual classifica os 
tipos de perda auditiva em condutiva, neurossensorial e mista. Segundo o grau da perda, os indivíduos foram classificados levando-se em conta a orelha de melhor audição, tal como proposto nos estudos de Lima, Aiello e Ferrari (23) e Costa, Sampaio e Oliveira (24). Utilizou-se a melhor orelha, pois a pior orelha tende a ser compensada pela função do melhor lado na percepção subjetiva (7). Essa classificação seguiu a recomendação da BIAP (Bureau Internacionald 'Audio Phonologie, 1997) (22), que utiliza a média aritmética das respostas nas frequências audiométricas de 500, 1000, 2000 e 4000Hz, e classifica os graus das perdas auditivas em: leve (de 21 a $40 \mathrm{dBNA}$ ); moderada de grau I (de 41-55dBNA); moderada de grau II (de 56-70dBNA); severa de grau I (de 71-80dBNA); severa de grau II (de 81-90dBNA); muito severa de grau I (de 91100dBNA); muito severa de grau II (de 101-110dBNA); muito severa de grau III (de 111-119dBNA) e perda auditiva total/cofose (a partir de 120dBNA).

A comparação dos resultados obtidos entre as duas formas de aplicação dos questionários (papel-lápis e frente a frente) foi realizada por meio do teste T de Student.Para a verificação de associação entre as variáveis utilizaram-se os testes estatísticos Qui-Quadrado e Exato de Fisher, sendo que este último foi utilizado como alternativa ao QuiQuadrado para o caso da amostra ser pequena em algumas células da tabela cruzada.

Para todos os testes acima citados, o nível de significância máximo assumido foi de $5 \%(\mathrm{p} \leq 0,05)$ e o softwareutilizado para a análise estatística foi o SPSS versão 10.0.

\section{RESULTADOS}

A amostra foi composta por 51 indivíduos, dos quais 49\% $(n=25)$ do sexo feminino e 51\% $(n=26)$ do sexo masculino. Do total de participantes do estudo, 31,3\% $(n=16)$ estavam na faixa etária entre 18 e 39 anos, 29,4\% $(n=15)$ estavam entre 40 e 59 , e 39,3\% $(n=20)$ tinham 60 anos ou mais. A idade média observada foi de 52 anos, com variação (desvio padrão) de 16,6 anos.

Quanto aos questionários, 60,7\% $(n=31)$ dos indivíduos responderam ao HHIA-S e 39,3\% $(n=20)$ responderam ao HHIE-S. Na comparação entre os questionários, não foi encontrada diferença estatisticamente significativa entre resultados dos mesmos $(t=0,22)$, mostrando que estes protocolos, mesmo em faixas etárias diferentes, podem ser aplicados com a mesma finalidade.

Com relação ao modo de aplicação dos questionários, 55\% $(n=28)$ dos indivíduos responderam no modo papel-lápis e $45 \%(n=23)$ responderam no modo frente a
Tabela I. Frequência dos diferentes graus de perda auditiva na orelha com melhor audição, encontrada nos participantes da amostra estudada.

\begin{tabular}{lrr}
\hline GraudaPerdaAuditiva & $\mathrm{n}$ & $(\%)$ \\
\hline Audição Normal & 16 & 31,30 \\
PerdaAuditiva Leve & 15 & 29,40 \\
PerdaAuditiva Moderada de Grau I & 8 & 15,60 \\
PerdaAuditiva Severa de Grau I & 2 & 3,90 \\
PerdaAuditiva exclusiva emFrequênciasAltas* & 10 & 19,60 \\
\hline Total & 51 & 100
\end{tabular}

* Classificação inexistente na proposta BIAP (1997): indica presença de audição média dentro da normalidade, mas presença de perda auditiva exclusiva em frequências altas.

frente. Também não houve diferença estatisticamente significativa na comparação entre os modos de aplicação dos questionários $(\mathrm{t}=0,16)$ de forma que os modos de aplicação foram agrupados para análise.

No que diz respeito ao tipo da perda auditiva, conforme a classificação de Silman e Silverman (21), 45\% $(n=23)$ da amostra apresentou perda auditiva neurossensorial, $11,7 \%(n=6)$ perda auditiva condutiva; $11,7 \%(n=6)$, perda auditiva mista, e 31,3\% $(n=16)$ apresentaram limiares auditivos normais.

Na Tabela 1, é apresentada a frequência dos diferentes graus de perda auditiva, levando-se em conta o grau da melhor orelha, ou seja, aquela que apresenta a melhor média audiométrica de acordo com os padrões sugeridos pela BIAP (21). Assim, foi observado que a maior parte dos indivíduos apresentou audição normal $(31,3 \%)$ e perda auditiva de grau leve (29,4\%).

No que se refere às respostas obtidas nos questionários, os indivíduos da amostra foram classificados de acordo com a proposta de Rosis, Souza e Iório (11), sendo obtidos os seguintes resultados: $29,4 \%(n=15)$ sem restrição de participação, 29,4\% $(n=15)$ com percepção de restrição de participação de leve a moderada e 41,1\% $(n=21)$ com percepção de restrição de participação significativa.

A avaliação audiológica convencional determinou o grau e o tipo da perda auditiva; os questionários a plicados avaliaram o grau de restrição de participação, ou seja, as desvantagens sociais e emocionais decorrentes da deficiência auditiva na amostra estudada. A partir disso, foram investigados os resultados da aplicabilidade dos instrumentos HHIA-S e HHIE-S na detecção da perda auditiva em pacientes adultos e idosos, além da capacidade dos mesmos em detectar diferentes tipos e graus de comprometimento auditivo. 
Tabela 2. Associação entre tipo de perda auditiva e grau da restrição de participação para a amostra estudada.

\begin{tabular}{|c|c|c|c|}
\hline \multirow[b]{2}{*}{ Tipo de perda } & \multicolumn{3}{|c|}{ Grau de restrição de participação } \\
\hline & $\begin{array}{c}\text { Sem } \\
\text { percepção }\end{array}$ & $\begin{array}{c}\text { Percepçãoleve } \\
\text { a moderada }\end{array}$ & $\begin{array}{l}\text { Percepçãa } \\
\text { significativa }\end{array}$ \\
\hline Limiares Auditiva normais & $46,7 \%$ & $23,1 \%$ & $26,1 \%$ \\
\hline PerdaAuditiva Condutiva & - & $15,4 \%$ & $17,4 \%$ \\
\hline PerdaAuditiva Mista & $6,7 \%$ & $30,8 \%$ & $4,3 \%$ \\
\hline PerdaAuditiva Neurossensorial & $46,7 \%$ & $30,8 \%$ & $52,2 \%$ \\
\hline$\overline{\text { Total }}$ & $100,0 \%$ & $100,0 \%$ & $100,0 \%$ \\
\hline
\end{tabular}

Tabela 3. Associação entre grau de perda auditiva e grau da restrição de participação para a amostra estudada.

\begin{tabular}{|c|c|c|c|}
\hline \multirow[b]{2}{*}{ Graude perda } & \multicolumn{3}{|c|}{ Grau de restrição de participação } \\
\hline & $\begin{array}{c}\text { Sem } \\
\text { percepção }\end{array}$ & $\begin{array}{c}\text { Percepção leve } \\
\text { a moderada }\end{array}$ & $\begin{array}{l}\text { Percepção } \\
\text { significativa }\end{array}$ \\
\hline $\begin{array}{l}\text { Audição Normal Bilateral } \\
\text { Audição Nomal Unilateral (com }\end{array}$ & $13,3 \%$ & $15,4 \%$ & $4,3 \%$ \\
\hline perda na outra orelha) & $33,3 \%$ & $7,7 \%$ & $21,7 \%$ \\
\hline PerdaAuditiva Leve & $20,0 \%$ & $30,8 \%$ & $34,8 \%$ \\
\hline PerdaAuditiva Moderada de Grau I & $6,7 \%$ & $23,1 \%$ & $17,4 \%$ \\
\hline PerdaAuditiva Severa de Grau I & $6,7 \%$ & $7,7 \%$ & -- \\
\hline PerdaAuditiva emfrequências altas & $20,0 \%$ & $15,4 \%$ & $21,7 \%$ \\
\hline Total & $100,0 \%$ & $100,0 \%$ & $100,0 \%$ \\
\hline
\end{tabular}

(Teste Exato de Fischer; $p=0.705)$.

Na Tabela 2, é mostrada a associação entre o tipo de perda auditiva e o grau de restrição de participação. Observa-se, nesta Tabela, que não existiu relação significativa $(p=0,701)$ entre qualquer tipo de perda auditiva e a presença de qualquer grau de restrição de participação, o que mostra que estes questionários não parecem ser instrumentos válidos para identificar diferentes tipos de perda auditiva.

Na Tabela 3, é apresentada a associação entre o grau da perda auditiva, de acordo com a classificação da BIAP (22), e o grau da restrição de participação na amostra estudada. Por meio do resultado do Teste Exato de Fisher, observa-se, mais uma vez nesta tabela, que não existe associação significativa entre o grau da perda auditiva e o grau de restrição de participação.

No Gráfico 1, é apontada a porcentagem de indivíduos com e sem perda auditiva e com e sem percepção de restrição de participação. Neste gráfico, é possível observar que $53,3 \%$ dos indivíduos com problema de audição, e $46,7 \%$ dos indivíduos sem problema auditivo não apresentam percepção de restrição de participação; por outro lado, $75 \%$ dos indivíduos com perda auditiva, e 25\% dos indivíduos sem perda apresentam percepção de restrição de

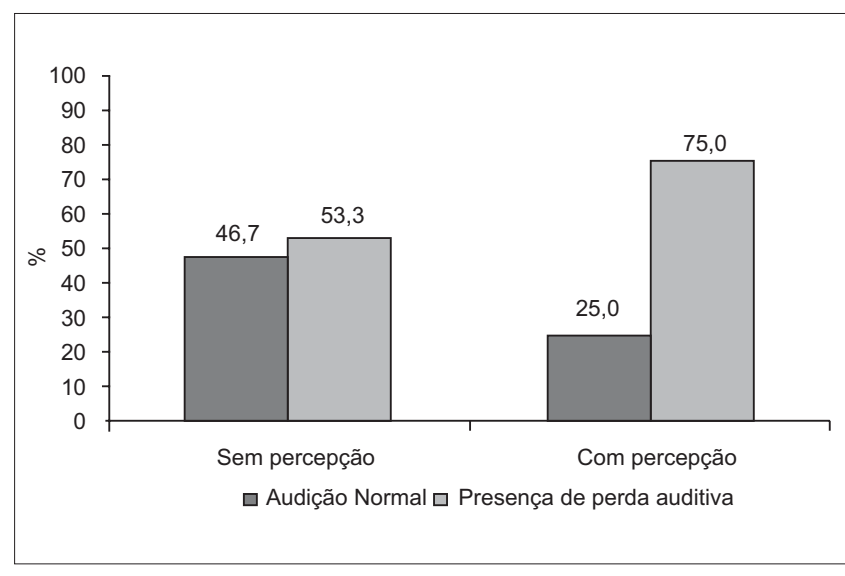

Gráfico 1. Porcentagem de indivíduos com e sem percepção de restrição de participação, segundo a presença ou não de perda auditiva, na amostra estudada (Teste Qui-Quadrado; $\mathrm{p}=0,118)$.

participação. Por meio do teste Qui-Quadrado, foi possível observar que não existiu associação significativa entre a presença ou não de perda auditiva de qualquer grau e a presença ou não de percepção de restrição de participação $(\mathrm{p}=0,118)$. 
Para analisar a validade dos questionários HHIE-S e HHIA-S quanto à sensibilidade e especificidade em termos de detecção de perdas auditivas e sua aplicabilidade em triagens auditivas, foram investigados quantos indivíduos eram corretamente detectados como tendo dificuldade auditiva, considerando que a precisão de um teste em identificar corretamente os pacientes positivos, ou seja, com distúrbio, é chamada sensibilidade e sua precisão em classificar corretamente os pacientes negativos, ou seja, sem distúrbio, é chamada especificidade (12).

Na amostra estudada, foi possível observar que dos 16 sujeitos com audição normal, 9 percebiam e 7 não percebiam restrição de participação decorrente de dificuldades auditivas; e que dos 35 participantes com perda auditiva, 27 apresentavam percepção de restrição de participação e 8 não percebiam a mesma. Com isso, os instrumentos revelaram baixa sensibilidade (47\%), ou seja, não identificaram indivíduos com perda auditiva; porém, apresentaram alta especificidade (75\%), identificando, corretamente, indivíduos que não apresentavam problemas de audição.

\section{DISCUSSÃO}

Os resultados obtidos no presente estudo demonstraram a presença de um número praticamente equivalente de mulheres e homens e um maior percentual de indivíduos adultos $(60,7 \%)$ do que idosos (39,3\%) na amostra analisada. Cabe ressaltar que esta amostra, de conveniência, foi composta por indivíduos que apresentavam queixas auditivas prévias, e que, portanto, haviam sido encaminhados para a avaliação audiológica. Assim, pode-se supor que indivíduos idosos sejam atendidos em outros centros de referência audiológica, que não no ambulatório onde a pesquisa foi realizada, uma vez que é esperado uma maior prevalência de perdas auditivas na terceira idade (25).

Na comparação entre os questionários, não houve diferença significativa entre resultados dos mesmos $(\mathrm{t}=$ $0,22)$. Esse dado era esperado, uma vez que Freitas e Costa (16) apontam que estes são os únicos protocolos equivalentes para serem aplicados em diferentes populações de acordo com a faixa etária. No que tange o modo de aplicação dos referidos instrumentos, não foi evidenciada diferença entre os dois tipos de aplicação, o que era esperado, pois, em outra pesquisa recente, o método de aplicação também não afetou o escore obtido em um questionário de autoavaliação relacionado à audição (26).

Conforme foi verificado, houve maior prevalência de perda auditiva neurossensorial na amostra estudada. Este resultado era previsto devido ao número de indivíduos idosos que constituíram a amostra, visto que nesta população é prevalente a ocorrência da presbiacusia (25). Esse achado também é compatível com o encontrado no estudo de JARDim et al (27), que do mesmo modo verificou um predomínio deste tipo de perda em indivíduos adultos e idosos atendidos no setor privado de um serviço de diagnóstico audiológicobrasileiro.

A frequência dos diferentes graus de perda auditiva, apresentados na Tabela 1 , apontou para um maior número de indivíduos com audição normal, embora um número destes tenha perda unilateral. Pode-se notar que, quanto maior a perda auditiva apresentada, menor o número de pessoas; o que pode ser explicado pelas perdas progressivas, caracterizadas pela presbiacusia (25), apresentadas pelos indivíduos idosos da amostra estudada.

No que diz respeito às respostas dos questionários, a maior parte dos indivíduos apresentaram restrição de participação significativa. Isso era esperado, pois a população envolvida na amostra apresentava queixas auditivas prévias e, portanto, referia dificuldade nas atividades diárias. Outro estudo realizado em São Paulo, também em um ambulatório de audiologia, encontrou resultados semelhantes nas porcentagens (12).

Na Tabela 2, não são observadas correlações estatisticamente significativas entre qualquer tipo de perda auditiva e grau de restrição de participação. Ainda nesta Tabela, é possível observar que 23,1\% e 26,1\% dos indivíduos com limiares auditivos normais a presentaram, respectivamente, percepção leve a moderada e percepção significativa do grau de restrição de participação. Este achado poderia ser explicado por alterações de processamento auditivo, pois alguns pacientes que apresentam audiometria dentro dos padrões de normalidade relatam queixas auditivas com relação à inteligibilidade de fala, em consequência de distúrbio do processamento auditivo (DPA), o que pode exercer efeito significativo sobre à autoavaliação da percepção de restrição de participação (26, 28, 29). Diante disso, foi levantada a hipótese de que estes indivíduos que não tinham perda auditiva periférica, mas que referiram alterações nos aspectos sociais e emocionais devido às dificuldades auditivas, possivelmente, apresentassem DPA.

A Tabela 3 mostra que não houve associação significativa entre grau da perda auditiva e grau de restrição de participação $(p=0,705)$. Esse achado concorda com a literatura nos trabalhos desenvolvidos por ArAújo et al. (30) e Rosis, Souza e Iório (12) que, também, observaram ausência entre esta relação, concluindo que a percepção do prejuízo auditivo não estava relacionada ao grau da perda auditiva.

Além disso, os resultados mostrados na Tabela 3 indicam que indivíduos com perda auditiva leve apresentaram maior grau de percepção de restrição de participação, 
o que revela que o grau da perda auditiva não é suficiente para evidenciar restrição das atividades diárias, pois indivíduos portadores de perda leve, moderada de grau I, severa de grau I e em frequências altas podem ter diferentes graus de percepção de restrição de participação. Este resultado também concorda com os achados de Correa e Russo (8), que verificaram, em sua pesquisa, indivíduos com perda leve ou moderada com maior grau de percepção de restrição de participação do que indivíduos com perdas maiores.

No Gráfico 1, é possível observar que 75\% dos indivíduos com percepção de restrição de participação apresentaram perda auditiva, e que 53,3\% dos que não referiram percepção dessa restrição também tinham perda de audição. Isso pode ter ocorrido pelo fato de muitos indivíduos terem respondidoao questionário de acordo com as adaptações já realizadas nas suas atividades diárias, conforme referido pelos mesmos às pesquisadoras durante a coleta. Por exemplo, na questão 8 do instrumento HHIAS ("Você tem dificuldade em escutar a TV ou o rádio por causa do problema de audição?"), alguns entrevistados responderam que não tinham dificuldades, pois bastava aumentar a intensidade do som dos a parelhos.

Este estudo mostrou que não existiu associação significativa entre a presença ou não de perda auditiva de qualquer grau e a presença ou não de percepção de restrição de participação $(p=0,118)$. Este achado corresponde ao de outro estudo (12), no qual também não foi encontrada associação estatística significativa entre o resultado encontrado na audiometria e a percepção de restrição de participação nos indivíduos atendidos no Ambulatóriode Audiologia da Universidade Federal de São Paulo (UNIFESP).

No que se refere à sensibilidade è̀ especificidade do uso dos questionários HHIA-S e HHIE-S na detecção de perda auditiva e sua aplicabilidade em triagem de adultos e idosos com problemas auditivos, o estudo apontou que estes instrumentos não parecem ser bons para detectar alterações de audição ou para triar indivíduos nos serviços de audiologia, nos quais os pacientes já chegam com queixas relacionadas à audição, acarretando em baixa sensibilidade (47\%) e alta especificidade (75\%). Esse achado concorda com outra pesquisa (12), que também aplicou o questionário HHIE-S e encontrou valores baixos para sensibilidade (23\%) e altos para especificidade (73,7\%), em grupo atendido em serviço de audiologia. Nesse mesmo estudo (12), para um grupo atendido em serviço de saúde não específico para atendimento relacionado às alterações auditivas, foram encontradas alta sensibilidade (94,7\%) e especificidade (75\%), mostrando que o questionário HHIE-S pode ser válido neste tipo de população como instrumento de triagem. Os achados da presente pesquisa também encontram apoio no trabalho realizado, no exterior, por GATEs et al. (31), que obteve resultados de 35\% e 94\% para sensibilidade especificidade, respectivamente, utilizando o instrumento HHIE-S em uma populaçãoidosa.

\section{CONCLUSÃO}

Na população estudada, os questionários HHIA-S e HHIE-S evidenciaram baixa sensibilidade e alta especificidade, demonstrando não serem instrumentos eficazes para triar indivíduos que já possuem queixas auditivas.

Ainda, na presente pesquisa, estes questionários não se mostraram eficientes para detectar diferentes tipos e graus de comprometimento auditivo, apontando que a percepção do comprometimento da audição não está relacionada necessariamente ao tipo ou ao grau da perda auditiva.

\section{REFERÊNCIAS BIBLIOGRÁFICAS}

1. Silva LPA, Queiros F, Lima I. Fatores etiológicos da deficiência auditiva em crianças e adolescentes de um centro de referência APADA em Salvador - BA. Rev Bras Otorrinolaringol. 2006, 72: 33-6.

2. Brasil. Ministério da Saúde. Secretaria de Atenção à Saúde. Portaria SAS/MS 587 de 7 de outubro de 2004 - Determina a organizaçãoe a implantação de Redes Estaduais de Atenção à Saúde Auditiva. Brasília (DF): Ministério da Saúde; 2004.

3. Mulrow CD, Aguilar C, Endicott JE, Velez R, Tuley MR, Charlip WS, etal. Association between hearing impairment and the quality of life of elderly individuals. J. Am. Geriatr. Soc. 1990, 38:45-50.

4. Cruz SM, Oliveira LR, Carandina L, Lima MCP, César CLG, Barros MBA et al.Prevalência de deficiência auditiva referida e causas atribuídas: um estudo de base populacional. Cad Saúde Pública. 2009, 25:1123-31.

5. Freitas KC, Calviti K, Pereira LD. Sensibilidade, Especificidade e Valores Preditivos Positivos da Queixa Auditiva Comparados com Diferentes Médias Audiométricas. Rev Bras Otorrinolaringol. 2001, 75:794-800.

6. Freitas CG. A Importância da Imitanciometria na Triagem Auditiva Pré-Escolar. Rio de Janeiro, 1999, p.22 (Monografia de Especialização em Audiologia Clínica .Centro de Especialização em Fonoaudiologia Clínica-CEFAC).

7. Rosalino CV, Rozenfeld S. Triagem auditiva em idosos: comparação entre auto-relato e audiometria. Rev Bras Otorrinolaringol. 2005, 71:193-200. 
8. Correa GF, Russo IC. Autopercepção do Handicap em Deficientes Auditivos Adultos e Idosos. Rev CEFAC. 1999, 1:54-63.

9. Macedo LS, Pupo C, Balieiro, CR. Aplicabilidade dos Questionários de Auto-avaliação em Adultos e Idosos com Deficiência Auditiva. Rev Dist Comum. 2006, 18:1925 .

10. Stewart M, Pankiw R, Lehman ME, Simpson TH Hearing Loss and Hearing Handicap in Users of Recreational Firearms. J Am Geriatr Soc. 2002, 13:160-8.

11. Chang HP, Ho CY, Chou P. The Factors Associated with a Self-Perceived Hearing Handicap im Elderly People with Hearing Impairment-Results from a Comunity - Based Study. Ear Hear. 2009, 30:576-583.

12. Rosis AC, Souza MR, Iório MC. Questionário Hearing Handicap Inventory for the Elderly - Screening Version (HHIE-S): estudo da sensibilidade e especificidade. Rev Soc Bras Fonoaudiol. 2009, 14:339-45.

13. Santiago LM, Novaes CD. Auto-Avaliação da Audição em Idosos. Rev CEFAC 2009, 11:98-105.

14. Calviti KCFK, Pereira LD. Sensibilidade, especificidade e valores preditivos da queixa auditiva comparados com diferentes médias audiométricas. Rev Bras Otorrinolaringol. 2009, 75:794-800.

15. Carvalho RD, Iório MC. Eficácia da Aplicação do Questionário de Handicap em Idosos Deficientes Auditivos. Rev Disturb Comum. 2007, 19:163-72.

16. Freitas CD, Costa MJ . Processo de Adaptação de Próteses Auditivas em Usuários Atendidos em uma Instituição Pública Federal - Parte II: resultados dos questionários de autoavaliação. Rev Bras Otorrinolaringol. 2007, 73:660-70.

17. Lautenschlager L, Tochetto T, Gonçalves MS, Barreto DCM, Moro MP, Bolzan GP. Percepção de Idosos Socialmente Ativos quanto às Desvantagens Causadas pela Dificuldade Auditiva. Rev Einstein. 2008, 6:155-58.

18. Teixeira AR, Freitas CLRF, MillãoLF, Gonçalves AK, Junior BB, Santos AMPV, et al. Relação entre a Queixa e a Presença de Perda Auditiva entre Idosos. Arq Int Otorrinolaringol. 2009, $13: 78-82$

19. Angeli RD, Jotz GP, Barba MC, Demeneghi PGM, Mello CHP. Efetividade de um Programa de Protetização Auditiva em Idosos Através da Aplicação do Questionário HHIE-S. Arq Int Otorrinolaringol. 2009, 13:277-80.
20. Marini ALS, Halpern R, Aerts D. Sensibilidade, Especificidade e Valor Preditivo da Queixa Auditiva. Rev Saúde Pública. 2005, 39:982-84.

21. Lei N. 8.842, de 4 de Janeiro de 1994. Ministério da Saúde.

22. Conselhos Federal e Regional de Fonoaudiologia. Orientações dos Conselhos de Fonoaudiologia para o Laudo Audiológico. 2009, 8-11.

23. Lima II, Aiello CP, Ferrari DV. Correlações Audiométricas do Questionário de HandicapAuditivo para Adultos. Rev CEFAC. 2010, 12:1-1.

24. Costa MHO, Sampaio ALL, Oliveira CACP. Avaliação do Benefício da Prótese Auditiva Digital e da Percepção da Desvantagem Auditiva ou "Handicap" em Idosos não Institucionalizados. Arq Int Otorrinolaringol. 2007, 11:15968

25. Nóbrega JD, Câmara MFS, Borges ACC. Audição do Idoso: Análise da Percepção do Prejuízo Auditivo, Perfil Audiológico e suas Correlações. Rev Bras Prom Saúde. 2008, 21:39-46.

26. Marotta RMB, Quintero, SM, Marone SAM. Avaliação do processamento auditivo por meio do teste de reconhecimento de dissílabos em tarefa dicótica SSW em indivíduos com audição normale ausência do reflexoacústico contralateral. Rev Bras Otorrinolaringol. 2002, 68:254-61.

27. Jardim IS, Iwahashi JH, Paula VC. Estudo do Perfil Audiológico de Indivíduos Atendidos em um Serviço de Diagnóstico Brasileiro. Arq Int Otorrinolaringol. 2010, 14: 32-37.

28. Quintero SM, Marotta RMB, Marone SAM. Avaliação do processamento auditivo em idosos com e sem presbiacusia por meio do teste de reconhecimento de dissílabos em tarefa dicótica - SSW. Rev Bras Otorrinolaringol. 2002, 68(1):2833.

29. Fire KM, Lesner SA, Newman C. Hearing handicap as a function of central auditory abilities in the elderly. Am J Otol. 1991, 12:105-08.

30. Araújo P G V, Mondelli MFCG, Lauris JRP, Richiéri AC, Feniman MR. Avaliação do handicap auditivo do adulto com deficiência auditiva unilateral. BrazJOtorhinolaryngol. 2010, $6: 370-83$.

31. Gates G A, Murphy M, Rees TS, Fraher A.Screening for handicapping hearing loss in the elderly. J F Pract. 2003, 52:56-62. 